\title{
Notes on van der Meer Scan for Absolute Luminosity Measurement
}

\author{
Vladislav Balagura ${ }^{a, b}$ \\ ${ }^{a}$ CERN, CH-1211 Geneve 23, Switzerland \\ ${ }^{b}$ ITEP, 117218, B.Cheremushkinskaya 25, Moscow, Russia
}

\begin{abstract}
An absolute luminosity can be measured in an accelerator by sweeping beams transversely across each other in the so called van der Meer scan. We prove that the method can be applied in the general case of arbitrary beam directions and a separation scan plane. A simple method to develop an image of the beam in its transverse plane from spatial distributions of interaction vertexes is also proposed. From the beam images one can determine their overlap and the absolute luminosity. This provides an alternative way of the luminosity measurement during van der Meer scan.
\end{abstract}

Keywords: luminosity, van der Meer scan, beam imaging

\section{Van der Meer method for arbitrary beam velocities}

The luminosity of an accelerator is given by

$$
L=f N_{1} N_{2} K \cdot \int \rho_{1}^{\mathrm{lab}}(\vec{r}-\Delta \vec{r}, t) \rho_{2}^{\mathrm{lab}}(\vec{r}, t) d^{3} \vec{r} d t
$$

where $K=\sqrt{\left(\vec{v}_{1}-\vec{v}_{2}\right)^{2}-\frac{\left(\vec{v}_{1} \times \vec{v}_{2}\right)^{2}}{c^{2}}}$ is the Møller kinematic relativistic factor [1], $c$ is the speed of light, $N_{1,2}$ are the number of particles in the colliding bunches all moving with the common velocities $\vec{v}_{1,2}, f$ is the frequency of collisions and $\rho_{1,2}^{\text {lab }}(\vec{r}, t)$ are the normalized particle densities in the laboratory frame, so that $\int \rho_{1,2}^{\mathrm{lab}}(\vec{r}, t) d^{3} \vec{r}=1$ at any time $t$. The absolute value of the luminosity or the cross section can be measured by separating the beams in the transverse plane by $\Delta \vec{r}$ and by monitoring a collision rate as a function of $\Delta \vec{r}$. This method was proposed by van der Meer more than 40 years ago and was originally proved in [2] for arbitrary beam shapes and parallel beams $\vec{v}_{1} \| \vec{v}_{2}$. It was successfully applied with various modifications at ISR [2, 3], RHIC [4] and recently at LHC [5] accelerators. It was often used in the approximation of Gaussian or double Gaussian beam shapes. At RHIC, for example, this allowed to take into account various corrections due to the so-called hourglass effect, the beam-beam deflection and the beam crossing angle. The latter alone, however, does not require any significant changes in the original van der Meer method. Since

Email address: balagura@cern.ch (Vladislav Balagura)

Preprint submitted to Elsevier

October 22, 2018 
we did not see any publication on this subject, in this section we present a proof of van der Meer formula in case of arbitrary beam crossing angle and beam shapes. It is applicable to the scans at LHC where hourglass and beam-beam effects are small [5].

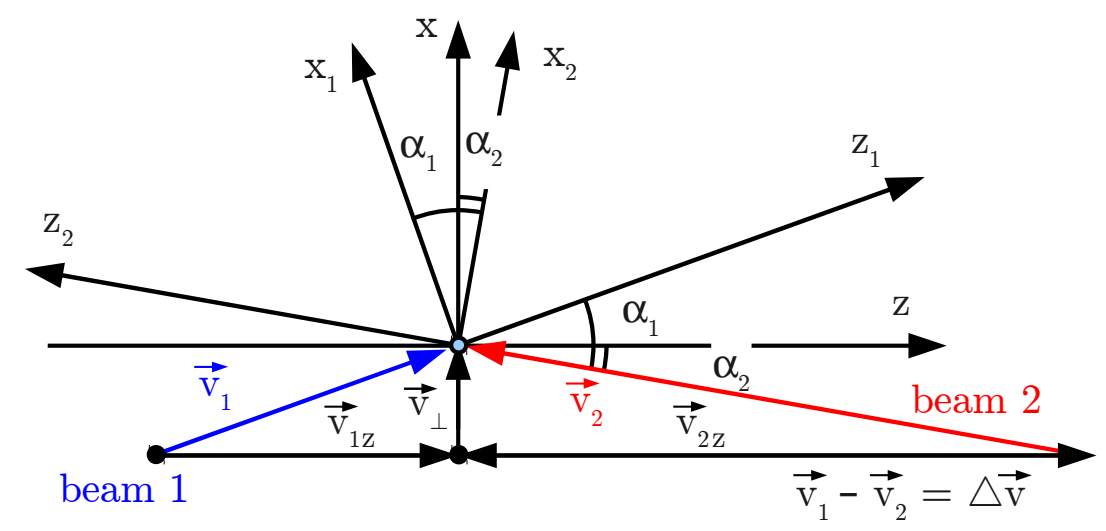

Figure 1: Laboratory $(x, z)$, first and second beam $\left(x_{1,2}, z_{1,2}\right)$ coordinate systems in the crossing plane.

Without loss of generality in Eq. (11) it is assumed that only the first beam is moved. We choose a coordinate system as shown in Fig. 1 with $z$ axis along the direction $\Delta \vec{v}=\vec{v}_{1}-\vec{v}_{2}$, $x$ axis lying in the beam crossing plane and $y$ axis perpendicular to $x$ and $z$. Let's denote $z$ - and $x$-components of the velocities as $\vec{v}_{1,2 z}$ and $\vec{v}_{\perp}$, respectively, so that $\vec{v}_{1,2}=\vec{v}_{\perp}+\vec{v}_{1,2 z}$. The beam displacement plane is not necessarily perpendicular to $z$, and in general $\Delta \vec{r}$ has three components $(\Delta x, \Delta y, \Delta z)$. Its projection to $x-y$ plane will be denoted by $\Delta \vec{r}_{\perp}$. For the particles uniformly moving with the velocities $\vec{v}_{1,2}$ the time evolution of their densities obeys the rule $\rho_{1,2}^{\text {lab }}(\vec{r}, t)=\rho_{1,2}^{\text {lab }}\left(\vec{r}-\vec{v}_{1,2} t, 0\right)$, therefore

$$
\begin{aligned}
\frac{L(\Delta \vec{r})}{f N_{1} N_{2} K}=\int \rho_{1}^{\mathrm{lab}}\left(\vec{r}-\Delta \vec{r}-\vec{v}_{\perp} t-\vec{v}_{1 z} t, 0\right) \cdot \rho_{2}^{\mathrm{lab}}\left(\vec{r}-\vec{v}_{\perp} t-\vec{v}_{2 z} t, 0\right) \frac{\partial(\vec{r}, t)}{\partial\left(\vec{r}-\vec{v}_{\perp} t, t\right)} d^{3}\left(\vec{r}-\vec{v}_{\perp} t\right) d t= \\
=\int\left[\int \rho_{1}^{\mathrm{lab}}\left(\vec{r}_{\perp}-\Delta \vec{r}_{\perp}, z^{\prime}, 0\right) d z^{\prime} \cdot \int \rho_{2}^{\mathrm{lab}}\left(\vec{r}_{\perp}, z^{\prime \prime}, 0\right) d z^{\prime \prime}\right] \frac{\partial(z, t)}{\partial\left(z^{\prime}, z^{\prime \prime}\right)} d^{2} \vec{r}_{\perp}= \\
=\frac{1}{|\Delta \vec{v}|} \int \rho_{1}^{\mathrm{lab}, \perp}\left(\vec{r}_{\perp}-\Delta \vec{r}_{\perp}\right) \rho_{2}^{\mathrm{lab}, \perp}\left(\vec{r}_{\perp}\right) d^{2} \vec{r}_{\perp}
\end{aligned}
$$

where we changed the integration variables to $\vec{r}-\vec{v}_{\perp} t=\left(x-v_{\perp} t, y, z\right)=\left(\vec{r}_{\perp}, z\right)$ and $z-\Delta z-v_{1 z} t=z^{\prime}, z+v_{2 z} t=z^{\prime \prime}$, the corresponding Jacobians were

$$
\frac{\partial(\vec{r}, t)}{\partial\left(\vec{r}-\vec{v}_{\perp} t, t\right)}=1, \quad \frac{\partial(z, t)}{\partial\left(z^{\prime}, z^{\prime \prime}\right)}=1 /|\Delta \vec{v}|
$$

We also used the notation $\rho_{1,2}^{\text {lab, } \perp}\left(\vec{r}_{\perp}\right)=\int \rho_{1,2}^{\text {lab }}\left(\vec{r}_{\perp}, z, 0\right) d z$ for the particle density projections on the plane perpendicular to $z \| \Delta \vec{v}$ at $t=0$. For the case $\vec{v}_{1} \| \vec{v}_{2}$ when $z \| \vec{v}_{1,2}$ we recover the usual formula

$$
L(\Delta \vec{r})=f N_{1} N_{2} \int \rho_{1}^{\mathrm{lab}, \perp}\left(\vec{r}_{\perp}-\Delta \vec{r}_{\perp}\right) \rho_{2}^{\mathrm{lab}, \perp}\left(\vec{r}_{\perp}\right) d^{2} \vec{r}_{\perp}
$$


Following van der Meer method, Eq. (2) should be integrated over $\Delta \vec{r}$. In threedimensional space an equation of $\Delta \vec{r}$ plane can be written in the form $\Delta \vec{r} \cdot \vec{n}=A$ where $A$ is some constant and $\vec{n}=\left(\cos \theta_{x}, \cos \theta_{y}, \cos \theta_{z}\right)$ is the unit normal, $\theta_{x, y, z}$ are the angles between $\vec{n}$ and the axes. Since Eq. (2) does not depend on $\Delta z$, an integration over $\Delta \vec{r}$ can be performed with the help of $\delta$-function as

$$
d^{2} \Delta \vec{r}=\delta(\Delta \vec{r} \cdot \vec{n}-A) d \Delta x d \Delta y d \Delta z=\frac{d \Delta x d \Delta y}{\cos \theta_{z}}=\frac{d^{2} \Delta \vec{r}_{\perp}}{\cos \theta_{z}} .
$$

In other words, the independence on $\Delta z$ allows to vary only $\Delta \vec{r}_{\perp}$ projection of $\Delta \vec{r}$, and $1 / \cos \theta_{z}$ appears as a difference between the area on $\Delta \vec{r}$ plane and its $x$ - $y$ projection. Integration of the density product in Eq. (2) over $d \Delta x d \Delta y=d^{2} \Delta \vec{r}_{\perp}$ gives

$$
\int \rho_{1}^{\text {lab }, \perp}\left(\vec{r}_{\perp}-\Delta \vec{r}_{\perp}\right) d^{2}\left(\vec{r}_{\perp}-\Delta \vec{r}_{\perp}\right) \times \int \rho_{2}^{\text {lab }, \perp}\left(\vec{r}_{\perp}\right) d^{2} \vec{r}_{\perp}=1
$$

and we obtain

$$
\int \frac{L(\Delta \vec{r})}{f N_{1} N_{2}} d^{2} \Delta \vec{r}=\frac{K}{\cos \theta_{z}|\Delta \vec{v}|}=\frac{1}{\cos \theta_{z}} \sqrt{1-\frac{\left(\vec{v}_{1} \times \vec{v}_{2}\right)^{2}}{\left(\vec{v}_{1}-\vec{v}_{2}\right)^{2} c^{2}}}
$$

For the process with the cross section $\sigma$ (including a reconstruction efficiency) the rate of events is given by

$$
R(\Delta \vec{r})=\sigma \cdot L(\Delta \vec{r})
$$

If $f, N_{1,2}$ are measured during the scan, the monitoring of the rate $R(\Delta \vec{r})$ allows to determine the cross section and then the absolute luminosity

$$
\sigma=\cos \theta_{z}\left[1-\frac{\left(\vec{v}_{1} \times \vec{v}_{2}\right)^{2}}{\left(\vec{v}_{1}-\vec{v}_{2}\right)^{2} c^{2}}\right]^{-1 / 2} \cdot \int \frac{R(\Delta \vec{r})}{f N_{1} N_{2}} d^{2} \Delta \vec{r} .
$$

This is the generalized van der Meer formula which is valid for any crossing angle between the beams and for arbitrary displacement plane. Note, that if the latter is not perpendicular to $z, \cos \theta_{z} \neq 1, \Delta z$ component of the displacement affects the time of the interactions but not the luminosity.

The term with the velocities $\vec{v}_{1,2}$ in Eq. (7) is simply a $\gamma$-factor of the boost with the velocity $\vec{v}_{\perp}$ (see Fig. 1). Indeed, it can be calculated as follows

$$
\begin{gathered}
v_{\perp}=v_{1} \sin \alpha_{1}=\frac{\left|\vec{v}_{1} \times\left(\vec{v}_{1}-\vec{v}_{2}\right)\right|}{\left|\vec{v}_{1}-\vec{v}_{2}\right|}=\frac{\left|\vec{v}_{1} \times \vec{v}_{2}\right|}{\left|\vec{v}_{1}-\vec{v}_{2}\right|}, \\
\gamma_{\perp}=\frac{1}{\sqrt{1-\left(v_{\perp} / c\right)^{2}}}=\left[1-\frac{\left(\vec{v}_{1} \times \vec{v}_{2}\right)^{2}}{\left(\vec{v}_{1}-\vec{v}_{2}\right)^{2} c^{2}}\right]^{-1 / 2}=\frac{|\Delta \vec{v}|}{K} .
\end{gathered}
$$

Its appearance here can be understood from the following alternative proof of Eq. (7). It is based on a relativistic invariance. We start from Eq. (3) used by van der Meer in his original paper [2] and valid for the collinear beams $\vec{v}_{1} \| \vec{v}_{2}$. Integration over $\Delta \vec{r}_{\perp}$ gives

$$
\sigma=\int \frac{(R / f)}{N_{1} N_{2}} d^{2} \Delta \vec{r}_{\perp}^{\text {coll }}
$$


where "coll" superscript of $\Delta \vec{r}_{\perp}$ coll reminds us that the formula is valid only in the frame where the beams are collinear. The element $\Delta \vec{r}_{\perp}$ coll is invariant only under boosts along $z$ which also preserve the condition $\vec{v}_{1} \| \vec{v}_{2}$. The ratio of two frequencies $R / f$ is an average number of interactions per collision, which does not depend on the choice of the coordinate system. $\sigma$ is also a relativistic invariant according to the cross section definition proposed by Møller [1]. The only non-invariant quantity in Eq. (9) is the displacement $\Delta \vec{r}_{\perp}^{\text {coll }}$.

The general case $\vec{v}_{1} \nVdash \vec{v}_{2}$ (see Fig. 1) can always be reduced to the collinear beams by making a boost with the velocity $\vec{v}_{\perp}$. It is easy to show that regardless of the laboratory $z$-components $\vec{v}_{1,2 z}$, in the relativistically boosted frame the beams become parallel to $z$, so that Eq. (9) is valid. To transform it back to the laboratory frame we note that $d^{2} \Delta \vec{r}_{\perp}$ transforms in the same way as a transverse part $d x d y$ of a relativistically invariant fourdimensional volume $d x d y d z d t$. Since $d z$ is not affected by the boost while $d t$ acquires a $\gamma$-factor, we have $d^{2} \Delta \vec{r}_{\perp}=d^{2} \Delta \vec{r}_{\perp}^{\text {coll }} / \gamma_{\perp}$, so that the relativistically invariant generalization of Eq. (9) is

$$
\sigma=\gamma_{\perp} \int \frac{R(\Delta \vec{r})}{f N_{1} N_{2}} d^{2} \Delta \vec{r}_{\perp}=\gamma_{\perp} \cos \theta_{z} \int \frac{R(\Delta \vec{r})}{f N_{1} N_{2}} d^{2} \Delta \vec{r}
$$

Together with Eq. (8) this completes the proof of Eq. (7).

For the following discussion it is useful to introduce coordinates linked to the beams as shown in Fig. 1. They are denoted by "1,2" subscripts. $z_{1,2}$ axes are chosen along the beams, $x_{1,2}$ axes lie in the beam crossing plane and other axes coincide, $y_{1}=y_{2}=y$. If $\alpha_{1,2}$ is the angle between $z_{1,2}$ and $z$, we have

$$
x=x_{1,2} \cos \alpha_{1,2}+z_{1,2} \sin \alpha_{1,2}, \quad z=\mp x_{1,2} \sin \alpha_{1,2} \pm z_{1,2} \cos \alpha_{1,2} .
$$

Let's assume that the distribution of particles in the transverse $x-y$ plane is independent in $x$ and $y$. In the beam's frame this means an independence in $x_{1,2} \cos \alpha_{1,2}+z_{1,2} \sin \alpha_{1,2}$ and $y$, which is usually ensured by an absence of $x_{1,2}-y$ accelerator coupling and an independence of longitudinal $\left(z_{1,2}\right)$ and transverse $(y)$ particle distributions. With this assumption, the two-dimensional integral over $\Delta \vec{r}_{\perp}$ can be reduced to the product of one-dimensional integrals along any $\Delta x=\Delta x_{0}$ and $\Delta y=\Delta y_{0}$ lines. Indeed, if the transverse densities factorize as $\rho_{1,2}^{\text {lab, } \perp}(x, y)=\rho_{1,2}^{\text {lab }} \frac{\perp}{x}(x) \cdot \rho_{1,2}^{\text {lab, }} \perp(y)$, the luminosity and the rate according to Eqs. (2) and (6) can also be factorized into $x$ - and $y$-dependent terms, $R(\Delta x, \Delta y)=R_{x}(\Delta x) \cdot R_{y}(\Delta y)$, and its integral over $d^{2} \Delta \vec{r}_{\perp}$ can be expressed as

$$
\begin{gathered}
\int R(\Delta x, \Delta y) d \Delta x d \Delta y=\int R_{x}(\Delta x) d \Delta x \int R_{y}(\Delta y) d \Delta y \frac{R_{x}\left(\Delta x_{0}\right) R_{y}\left(\Delta y_{0}\right)}{R\left(\Delta x_{0}, \Delta y_{0}\right)}= \\
=\frac{\int R\left(\Delta x, \Delta y_{0}\right) d \Delta x \cdot \int R\left(\Delta x_{0}, \Delta y\right) d \Delta y}{R\left(\Delta x_{0}, \Delta y_{0}\right)} .
\end{gathered}
$$

In Eq. (12) we considered the simple case when the integration is performed over $\Delta x-\Delta y$ plane, so that $\cos \theta_{z}=1$. After substituting Eq. (12) into (10), the cross section can be written as

$$
\sigma=\gamma_{\perp} \frac{\int R\left(\Delta x, \Delta y_{0}\right) d \Delta x \cdot \int R\left(\Delta x_{0}, \Delta y\right) d \Delta y}{f N_{1} N_{2} R\left(\Delta x_{0}, \Delta y_{0}\right)} .
$$


The integrals in the enumerator of Eq. (12) can be measured in two one-dimensional scans over $\Delta x$ at fixed $\Delta y_{0}$ and vice versa. There is no need to make a full scan in $\Delta x-\Delta y$ plane. Note, that to get larger rates it is advantageous to keep the beam separation $\left(\Delta x_{0}, \Delta y_{0}\right)$ small, but in general Eq. (13) is valid for arbitrary $\left(\Delta x_{0}, \Delta y_{0}\right)$.

In derivation of Eq. 13 we do not use the fact that $x$ axis lies in the crossing plane. If the transverse distributions $\rho_{1,2}^{\text {lab, } \perp}$ are independent in two arbitrary directions $x^{\prime}$ and $y^{\prime}$ in $\Delta \vec{r}_{\perp}$ plane, the formula (13) written in the primed coordinates still remains valid, and $x^{\prime}$ and $y^{\prime}$ may be chosen as scan axes. If they are not perpendicular but form an angle $\alpha_{x^{\prime} y^{\prime}}$, one should also include the corresponding Jacobian $\sin \alpha_{x^{\prime} y^{\prime}}$. Finally, the two scan axes may extend beyond the $x-y$ plane if their projections still coincide with $x^{\prime}, y^{\prime}$ axes. Let's denote the displacements along such scan axes as $\Delta x^{\prime \prime}, \Delta y^{\prime \prime}$ and their inclination angles to $x-y$ plane as $\alpha_{x^{\prime \prime}, y^{\prime \prime}}$. Taking into account that $d \Delta x^{\prime}\left(y^{\prime}\right)=\cos \alpha_{x^{\prime \prime}\left(y^{\prime \prime}\right)} d \Delta x^{\prime \prime}\left(y^{\prime \prime}\right)$, one obtains a more general formula

$$
\sigma=\gamma_{\perp} \sin \alpha_{x^{\prime} y^{\prime}} \cos \alpha_{x^{\prime \prime}} \cos \alpha_{y^{\prime \prime}} \frac{\int R\left(\Delta x^{\prime \prime}, \Delta y_{0}^{\prime \prime}\right) d \Delta x^{\prime \prime} \cdot \int R\left(\Delta x_{0}^{\prime \prime}, \Delta y^{\prime \prime}\right) d \Delta y^{\prime \prime}}{f N_{1} N_{2} R\left(\Delta x_{0}^{\prime \prime}, \Delta y_{0}^{\prime \prime}\right)}
$$

\section{Reconstruction of individual beam profiles}

The density of interaction vertexes accumulated during time $\Delta T$ is given by

$$
\frac{d^{3} N_{\mathrm{vx}}}{d x d y d z}=f \Delta T N_{1} N_{2} K \sigma \int \rho_{1}^{\mathrm{lab}}(\vec{r}-\delta \vec{r}-\Delta \vec{r}, t) \rho_{2}^{\mathrm{lab}}(\vec{r}-\delta \vec{r}, t) V^{\mathrm{lab}}(\delta \vec{r}) d t d^{3} \delta \vec{r} .
$$

This expression is very similar to the luminosity formula (1) except it contains the convolution with the experimental vertex resolution $V^{\text {lab }}(\delta \vec{r})$ and there is no integration over $d^{3} \vec{r}$. In analogy with van der Meer method, we may integrate this equation over $d^{2} \Delta \vec{r}$ to drop out $\rho_{1}^{\text {lab }}$, and after deconvolution with $V^{\text {lab }}(\delta \vec{r})$ obtain the profile of the second beam $\rho_{2}^{\text {lab }}$. A possible non-zero beam crossing angle complicates the formulas, but as it is shown below, it is always possible to reconstruct the profiles of both beams in their transverse planes without any simplifying assumptions on the beam shapes.

It is convenient to express the particle density of the second beam in its own coordinate system $\left(x_{2}, y, z_{2}\right)=\vec{r}_{2}$ (see Fig. 11) as

$$
\rho_{2}\left(\vec{r}_{2}, t\right)=\rho_{2}^{\mathrm{lab}}(\vec{r}, t)=\rho_{2}\left(\vec{r}_{2}^{\perp}, z_{2}-\left|\vec{v}_{2}\right| t, 0\right) .
$$

The transformation Eqs. (11) define the relation between the densities $\rho_{2}^{\text {lab }}$ and $\rho_{2}$ in the laboratory and in the second beam frames, respectively. In the last equation we distinguished transverse $\vec{r}_{2}^{\perp}=\left(x_{2}, y\right)$ and longitudinal $z_{2}$ coordinates and took into account that $z_{2} \| \vec{v}_{2}$. In addition, let's define the resolution viewed from the second beam frame as

$$
V_{2}\left(\delta \vec{r}_{2}\right)=V^{\mathrm{lab}}(\delta \vec{r})
$$

For the first beam we define $\vec{R}=\vec{r}-\Delta \vec{r}-\vec{v}_{1} t$, so that

$$
\rho_{1}^{\mathrm{lab}}(\vec{r}-\delta \vec{r}-\Delta \vec{r}, t)=\rho_{1}^{\mathrm{lab}}(\vec{R}-\delta \vec{r}, 0) .
$$


Since $\int \rho_{1}^{\text {lab }}(\vec{R}-\delta \vec{r}, 0) d^{3} \vec{R}=1$, to drop out $\rho_{1}^{\text {lab }}$ from Eq. (15) one needs a three-dimensional integration over $\vec{R}$. The volume element $d^{3} \vec{R}$ is invariant under rotations. The simplest is to write it in the coordinate system with $x$ and $y$ axes lying in the $\Delta \vec{r}$ displacement plane and with $z$ axis pointing along the unit normal $\vec{n}$. In components, $d^{3} \vec{R}=d X^{\Delta} d Y^{\Delta} d Z^{\Delta}$, where $\Delta$ superscript denotes coordinates in this system. Only $\rho_{1}^{\text {lab }}$ in Eq. (15) depends on $\Delta \vec{r}$, therefore regardless of other variables, integration over $\Delta \vec{r}$ is equivalent to integration of $\rho_{1}^{\text {lab }}$ over $d X^{\Delta} d Y^{\Delta}$. The third coordinate $Z^{\Delta}=\vec{R} \cdot \vec{n}$ is spanned when integrating over $t$ due to $\left|\vec{v}_{1}\right| t$ term in $\vec{R}$.

The density of the second beam, $\rho_{2}\left(\vec{r}_{2}^{\perp}, z_{2}-\left|\vec{v}_{2}\right| t, 0\right)$ also depends on $t$, however. To decouple $\rho_{1}^{\text {lab }}$ and $\rho_{2}$, one may integrate in addition over $z_{2}$ of the reconstructed vertexes. Then it is possible to change the integration variables from $d t d z_{2}$ to $d Z^{\Delta} d Z_{2}$, where $Z_{2}=$ $z_{2}-\left|\vec{v}_{2}\right| t . d Z^{\Delta}$ completes the integration over $d^{3} \vec{R}$, while the integration over $d Z_{2}$ gives a projection transverse to the second beam

$$
\rho_{2}^{\perp}\left(\vec{r}_{2}^{\perp}\right)=\int \rho_{2}\left(\vec{r}_{2}^{\perp}, Z_{2}-\delta z_{2}, 0\right) d Z_{2}=\int \rho_{2}\left(\vec{r}_{2}^{\perp}, z, 0\right) d z
$$

Since $\vec{r}$ in $\vec{R}$ also depends on $z_{2}$ and $\partial \vec{r} / \partial z_{2}=\vec{v}_{2} /\left|\vec{v}_{2}\right|$, the Jacobian of the variables substitution is

$$
\left|\frac{\partial\left(t, z_{2}\right)}{\partial\left(Z^{\Delta}, Z_{2}\right)}\right|=\frac{1}{\left|\vec{n} \cdot\left(\vec{v}_{1}-\vec{v}_{2}\right)\right|}=\frac{1}{\cos \theta_{z} \cdot|\Delta \vec{v}|}
$$

where $\cos \theta_{z}$ is the same angle as in Eq. (5) between $\vec{n}$ and $z \|\left(\vec{v}_{1}-\vec{v}_{2}\right)$.

Combining all pieces (15)-(20) and (8) together, one has

$$
\begin{gathered}
\gamma_{\perp} \cos \theta_{z} \int \frac{1}{f \Delta T N_{1} N_{2} \sigma} \cdot \frac{d^{3} N_{\mathrm{vx}}}{d x_{2} d y_{2} d z_{2}} d z_{2} d^{2} \Delta \vec{r}=\int \rho_{1}^{\mathrm{lab}}(\vec{R}-\delta \vec{r}, 0) d^{3} \vec{R} \times \\
\times \int \rho_{2}\left(\vec{r}_{2}^{\perp}-\delta \vec{r}_{2}^{\perp}, Z_{2}-\delta z_{2}, 0\right) d Z_{2} \int V_{2}\left(\delta \vec{r}_{2}^{\perp}, \delta z_{2}\right) d \delta z_{2} d^{2} \delta \vec{r}_{2}^{\perp}= \\
=\int \rho_{2}^{\perp}\left(\vec{r}_{2}^{\perp}-\delta \vec{r}_{2}^{\perp}\right) V_{2}^{\perp}\left(\delta \vec{r}_{2}^{\perp}\right) d^{2} \delta \vec{r}_{2}^{\perp} .
\end{gathered}
$$

Here were used the rotation invariance of the volume element $d x d y d z=d x_{2} d y_{2} d z_{2}$ and the same for $d^{3} \delta \vec{r}$, and also defined

$$
V_{2}^{\perp}\left(\delta \vec{r}_{2}^{\perp}\right)=\int V_{2}\left(\delta \vec{r}_{2}^{\perp}, \delta z_{2}\right) d \delta z_{2}=\int V^{\mathrm{lab}}(\delta \vec{r}) d \delta z_{2}
$$

which is the vertex resolution in the beam transverse plane. Integration of Eq. (21) over $\vec{r}_{2}^{\perp}$ gives the total number of interactions and their rate consistent with Eq. (10). Unfolding Eq. (21) with $V_{2}^{\perp}\left(\delta \vec{r}_{2}^{\perp}\right)$ gives the image of the second beam in its transverse plane $\rho_{2}^{\perp}\left(\vec{r}_{2}^{\perp}\right)$.

From the very beginning it was assumed that only the first beam is moved in van der Meer scan. Instead, one can go to its "rest" frame where vice versa, only the second beam moves 
by $-\Delta \vec{r}$ and the first is stable. By repeating the procedure above, one can then measure the transverse image of the first beam, as in this case the integration over $\Delta \vec{r}$ and $z_{1}$ leads to the same Eq. (21) with the index 2 substituted by 1. It is interesting that two beam images can be obtained simultaneously from the same set of vertex distributions measured at various $\Delta \vec{r}$ points. They should be brought to the rest frame of the corresponding beam, i.e. aligned differently during summation, and projected to the beam transverse plane.

If $f, \Delta T, N_{1,2}$ or the reconstruction efficiency entering $\sigma$ change during the scan, they should be considered as functions of $\Delta \vec{r}$ during the integration on the left side of Eq. (21). On practice, this means that the accumulated vertex distributions and their statistical errors should be reweighted according to the factor $\left(f \Delta T N_{1} N_{2} \sigma\right)^{-1}$ at every scan point.

One can determine not only transverse but the full three-dimensional images $\rho_{1,2}$, if the detector is able to measure the time $t$ of the interaction. Indeed, in this case Eq. (15) may be integrated over $\Delta \vec{r}$ and $\left(\vec{r}-\vec{v}_{1} t\right) \cdot \vec{n}=Z^{\Delta}$, while the integration over $Z_{2}=z_{2}-\left|\vec{v}_{2}\right| t$ may be omitted. This is possible since $Z^{\Delta}$ is directly measured. In addition to the vertex resolution $V^{\text {lab }}(\delta \vec{r})$, we should include in the formulas the time resolution $V_{t}(\delta t)$, make a change $t \rightarrow t-\delta t$ and integrate over $\delta t$. As before, after the integration over $d^{2} \Delta \vec{r} d Z^{\Delta}$ or $d^{3} \vec{R}$ the density $\rho_{1}^{\text {lab }}$ drops out and we obtain

$$
\begin{gathered}
\gamma_{\perp} \cos \theta_{z} \int \frac{1}{f \Delta T N_{1} N_{2} \sigma} \cdot \frac{d^{4} N_{\mathrm{vx}}}{d x d y d z d t} d^{2} \Delta \vec{r} d\left(\left[\vec{r}-\vec{v}_{1} t\right] \cdot \vec{n}\right)= \\
=\int \rho_{2}^{\mathrm{lab}}\left(\vec{r}-\delta \vec{r}-\vec{v}_{2}(t-\delta t), 0\right) V^{\mathrm{lab}}(\delta \vec{r}) V_{t}(\delta t) d^{3} \delta \vec{r} d \delta t .
\end{gathered}
$$

The distribution of vertexes in $\left(x_{2}, y_{2}, z_{2}-\left|\vec{v}_{2}\right| t\right)$ space, accumulated during the scan and deconvolved with the resolutions determine the density $\rho_{2}$. Note, that the time resolution affects the imaging in the longitudinal $z_{2} \| \vec{v}_{2}$ direction. With an infinitely poor resolution in $z_{2}-\left|\overrightarrow{v_{2}}\right| t$ measurement, Eq. (23) becomes effectively equivalent to Eq. (21).

Now let's consider the case when the particle distributions in $y$ direction and in $x-z$ crossing plane are independent. In the following, $x, z$ - and $y$-dependent parts of a function will be denoted by the corresponding subscripts, so that we have $\rho_{1,2}^{\text {lab }}(x, y, z, 0)=$ $\rho_{1,2 x z}^{\text {lab }}(x, z) \cdot \rho_{1,2}^{\text {lab }}(y)$. As it was discussed in Sec. 1, instead of scanning the full $\Delta \vec{r}$ plane in this case it was sufficient to make two one-dimensional scans over $\Delta x$ and $\Delta y$. Instead of $\Delta x$, any other line in $x-z$ plane may be taken, so for generality we consider the line inclined at an angle $\theta_{z}$ from the $x$ axis and denote the corresponding displacement as $\Delta \vec{r}_{x z}=\left(\Delta r_{x z} \cdot \cos \theta_{z}, 0, \Delta r_{x z} \cdot \sin \theta_{z}\right)$.

If the spatial resolution also factorizes, $V^{\mathrm{lab}}(\delta \vec{r})=V_{x z}^{\mathrm{lab}}(\delta x, \delta z) \cdot V_{y}^{\mathrm{lab}}(\delta y)$, according to Eq. (15) the vertex density can be split into two parts:

$$
\frac{\gamma_{\perp} \cos \theta_{z}}{f \Delta T N_{1} N_{2} \sigma} \frac{d^{3} N_{\mathrm{vx}}}{d x d y d z}=\rho^{\mathrm{Vx}}(\vec{r}, \Delta \vec{r})=\rho_{x z}^{\mathrm{vx}}\left(x, z, \Delta r_{x z}\right) \cdot \rho_{y}^{\mathrm{vx}}(y, \Delta y) .
$$

$\rho^{\mathrm{VX}}$ is the overlap integral from Eq. (15), its normalization follows from Eq. (10)

$$
\int \rho^{\mathrm{VX}}(\vec{r}, \Delta \vec{r}) d^{3} \vec{r} d^{2} \Delta \vec{r}=1
$$


Similarly, the relative normalization of $\rho_{x z}^{\mathrm{VX}}$ and $\rho_{y}^{\mathrm{VX}}$ may be fixed by the requirement

$$
\int \rho_{x z}^{\mathrm{vx}}\left(x, z, \Delta r_{x z}\right) d x d z d \Delta r_{x z}=\int \rho_{y}^{\mathrm{vx}}(y, \Delta y) d y d \Delta y=1 .
$$

Following the same arguments as above one may then obtain the analogs of Eq. (21):

$$
\begin{gathered}
\int \rho_{x z}^{\mathrm{Vx}}\left(x, z, \Delta r_{x z}\right) d z_{2} d \Delta r_{x z}=\int \rho_{2 x 2}\left(x_{2}-\delta x_{2}\right) V_{x 2}\left(\delta x_{2}\right) d \delta x_{2}, \\
\int \rho_{y}^{\mathrm{VX}}(y, \Delta y) d \Delta y=\int \rho_{2 y}^{\mathrm{lab}}(y-\delta y) V_{y}^{\mathrm{lab}}(\delta y) d \delta y,
\end{gathered}
$$

where $\rho_{2 x 2}\left(\rho_{2 y}^{\text {lab }}\right), V_{x 2}\left(V_{y}^{\text {lab }}\right)$ are the second beam transverse profile along $x_{2}(y)$ and the corresponding projection of the resolution,

$$
\rho_{2 x 2}\left(x_{2}\right)=\int \rho_{2 x z}^{\mathrm{lab}}(x, z) d z_{2}, \quad V_{x 2}\left(\delta x_{2}\right)=\int V_{x z}^{\mathrm{lab}}(\delta x, \delta z) d \delta z_{2} .
$$

If one integrates Eq. (24) over $d^{3} \vec{r}$, all information about the spatial distribution of vertexes is lost and the result should be expressible via rates. To be consistent with the normalization conditions (26) one should choose

$$
\begin{gathered}
\int \rho_{x z}^{\mathrm{vx}}\left(x, z, \Delta r_{x z}^{0}\right) d x d z=\frac{R\left(\Delta r_{x z}^{0}, \Delta y_{0}\right)}{\int R\left(\Delta r_{x z}, \Delta y_{0}\right) d \Delta r_{x z}}, \\
\int \rho_{y}^{\mathrm{Vx}}\left(y, \Delta y_{0}\right) d y=\frac{R\left(\Delta r_{x z}^{0}, \Delta y_{0}\right)}{\int R\left(\Delta r_{x z}^{0}, \Delta y\right) d \Delta y},
\end{gathered}
$$

where $\Delta r_{x z}^{0}$ and $\Delta y_{0}$ are the fixed displacements during the scan performed along the other coordinate. As it was pointed out in Sec. 1, they may be arbitrary. Indeed, since the rate is factorisable, $\Delta r_{x z}^{0}$ or $\Delta y_{0}$ appearing both in enumerator and in denominator cancel out. Note, that both Eqs. (27) and (29) lead to (26) after integration over $x_{2}, y$, or over $\Delta r_{x z}^{0}$, $\Delta y_{0}$, respectively.

Taking into account Eqs. (27) and (29), one can integrate (24) over $d y d z_{2} d \Delta r_{x z}$ or over $d x d z d \Delta y$ and finally obtain

$$
\begin{aligned}
& \int \frac{d N_{\mathrm{VX}}\left(\Delta r_{x z}, \Delta y_{0}\right)}{d x_{2}} \frac{d \Delta r_{x z}}{N_{0}}=\frac{R\left(\Delta r_{x z}^{0}, \Delta y_{0}\right)}{\int R\left(\Delta r_{x z}^{0}, \Delta y\right) d \Delta y} \int \rho_{2} x_{2}\left(x_{2}-\delta x_{2}\right) V_{x 2}\left(\delta x_{2}\right) d \delta x_{2}, \\
& \int \frac{d N_{\mathrm{VX}}\left(\Delta r_{x z}^{0}, \Delta y\right)}{d y} \frac{d \Delta y}{N_{0}}=\frac{R\left(\Delta r_{x z}^{0}, \Delta y_{0}\right)}{\int R\left(\Delta r_{x z}, \Delta y_{0}\right) d \Delta r_{x z}} \int \rho_{2}^{\mathrm{lab}}(y-\delta y) V_{y}^{\mathrm{lab}}(\delta y) d \delta y,
\end{aligned}
$$

where $N_{0}=\frac{f \Delta T N_{1} N_{2} \sigma}{\gamma_{\perp} \cos \theta_{z}}=\int N_{\mathrm{VX}}\left(\Delta r_{x z}, \Delta y\right) d \Delta r_{x z} d \Delta y$. One can see that the distribution of vertexes accumulated during $\Delta r_{x z}(\Delta y)$ scan, projected to $x_{2}(y)$ axis, normalised and unfolded with the corresponding projection of the resolution $V_{x 2}\left(V_{y}^{\text {lab }}\right)$, gives the transverse beam profile $\rho_{2} x_{x 2}\left(x_{2}\right)\left(\rho_{2}^{\text {lab }}(y)\right)$.

If the scan is performed not continuously but stepwise, so that the data is taken at discrete points $\Delta x= \pm n \epsilon_{x}, \Delta y= \pm m \epsilon_{y}$ with some integers $n$ and $m$, the integrations may be approximated as discrete sums. This brings some systematic uncertainty, which may be estimated in the end from the reconstructed beam images. 


\section{Discussion and conclusions}

The collider luminosity and cross sections can be measured in van der Meer scan by sweeping the beams transversely across each other and by measuring the collision rate as a function of the beam displacement. We proved that the method remains applicable for the arbitrary beam crossing angle and the beam displacement plane, see Eqs. (10), (13) and (14).

The four-dimensional beam densities may also be arbitrary. In particular, the formulas are valid for the beams initially mismatched in time. With non-zero crossing angle the time shift between the beams is equivalent to some shift in $\Delta x$, and therefore the integration over $\Delta x$ in van der Meer method removes the dependence on timing. Also note, that in the case of $x-y$ factorization, when it is sufficient to scan only along two perpendicular axes, their crossing point $\left(\Delta x_{0}, \Delta y_{0}\right)$ in Eq. (13) is also arbitrary and not necessarily the point of maximal luminosity. The beams at this point can be mismatched in $\Delta x$ or, equivalently, in time.

The results are applicable to van der Meer scans performed at LHC accelerator in 2010. This was a primary tool for the absolute luminosity measurement at four major LHC experiments. The maximal crossing angle of $540 \mu \mathrm{rad}$ was in LHCb. It reduced the luminosity by about $4 \%$, but caused a negligible correction, $\gamma_{\perp}-1=4 \cdot 10^{-8}$, in van der Meer formula (13).

The original van der Meer method suggested in 1968 was based on counting the interactions. An important information is also contained in the spatial distribution of vertexes. With excellent modern detectors, like in LHC experiments, they are precisely measurable. We propose a new simple method how they can be used to reconstruct the beam images. The vertex distributions transverse to the beam and visible from its center should be accumulated during the scan and unfolded with the transverse vertex resolution. This should give the beam image in its transverse plane, see Eqs. (21) and (30). The approach is valid for arbitrary beam shapes.

From the reconstructed normalized beam profiles one can determine their overlap and then the luminosity using Eq. (2). This gives an alternative way of the absolute luminosity measurement during van der Meer scan. Two methods are independent as the beam imaging method uses only the normalized shapes of the accumulated vertex distributions, while the traditional method uses only the integrals, i.e. the total number of interactions.

There is one complication in determining the luminosity from the images, however. According to Eq. (2), the luminosity depends on the overlap integral of the beam profiles in $x$-y laboratory plane, $\rho_{1,2}^{\text {lab, } \perp}\left(\vec{r}_{\perp}\right)$. With the proposed imaging method one can reconstruct the profiles $\rho_{1,2}^{\perp}\left(\vec{r}_{1,2}^{\perp}\right)$ transverse to the beams, see Eq. (21). They coincide with $\rho_{1,2}^{\text {lab, } \perp}\left(\vec{r}_{\perp}\right)$ only for the collinear beams, but in general lead to a different overlap integral.

To correct for this effect, some information is needed on the distribution of particles along the beam. For example, in LHC one can do the following. As it was pointed out, the non-zero crossing angle reduced the luminosity and the overlap integral in 2010 scans by $4 \%$ or less. Since the effect is small, for its estimation it may be sufficient to approximate the bunch shapes along $x_{1,2}$ and $z_{1,2}$ as independent Gaussians with some effective 
sigmas $\sigma_{x 1,2}$ and $\sigma_{z 1,2}$. In this case the $x$-projections in the laboratory frame are also Gaussian with sigmas $\sigma_{1,2}^{\text {lab } x}=\sqrt{\left(\sigma_{x 1,2} \cos \alpha_{1,2}\right)^{2}+\left(\sigma_{z 1,2} \sin \alpha_{1,2}\right)^{2}}$. If the bunch lengths of two beams are similar, $\sigma_{z 1} \approx \sigma_{z 2}$, they can be obtained from the $z$-width of the luminous region $\sigma_{z 1,2} \approx \sqrt{2} \sigma_{\text {lum }}^{z}$. Here we assumed collinear beams, but corrections due to non-zero crossing angle are negligible since in LHC $\cos \alpha_{1,2} \approx 1$ and transverse sizes of the bunches are about three orders of magnitude smaller than their lengths. Since for Gaussian beams the luminosity is proportional to $\sqrt{\sigma_{x 1}^{2}+\sigma_{x 2}^{2}}$, its reduction due to the crossing angle is $\sqrt{\sigma_{x 1}^{2}+\sigma_{x 2}^{2}} / \sqrt{\left(\sigma_{x 1} \cos \alpha_{1}\right)^{2}+\left(\sigma_{x 2} \cos \alpha_{2}\right)^{2}+2\left(\sigma_{\text {lum }}^{z}\right)^{2}\left(\sin ^{2} \alpha_{1}+\sin ^{2} \alpha_{2}\right)}$.

If the detector is able to measure both the spatial coordinates and the time of the interactions, one can reconstruct not only transverse but the full three-dimensional beam images, see Eq. (23). The luminosity can be determined from them without any extra corrections or assumptions.

The proposed imaging during van der Meer scan is very similar to the beam-gas imaging [6]. The idea of the latter is to take a beam "photo" using the interactions with the gas remaining in the beam pipe, assuming it is distributed uniformly in the transverse plane. In both methods, after deconvolution with the vertex resolution, one can measure the transverse beam profiles and then the luminosity, taking into account the crossing angle correction. The beam-gas imaging method was successfully applied for the first time to measure the absolute luminosity in LHCb [7]. Its accuracy, as in van der Meer LHC scans in 2010, was dominated by errors in bunch intensity $N_{1,2}$ measurement. The beam-gas method does not require moving of the beams and can be used during normal data taking. On the other hand, the advantage of the beam imaging during van der Meer scan is a much higher statistics of interactions and a localization of vertexes around a nominal luminous region where the vertex resolution is optimal. The methods have different systematics, and it is very advantageous to use both during van der Meer scan.

\section{References}

[1] C.Møller, K. Danske Vidensk. Selsk. Mat.-Fys. Medd. 23 (1945) 1; for later papers see e.g. O. Napoly, Part. Acc. 40 (1993) 181; W.Herr, B.Muratory, "Concept of Luminosity", Proceedings of CERN Accelerator School, (2003) 361.

[2] Van der Meer, "Calibration of the Effective Beam Height in the ISR", internal CERN report, ISRPO/68-31 (1968).

[3] K. Potter and S. Turner, IEEE Trans. Nucl. Sci. 22 (1975) 1589;

P. Bryant and K. Potter, "Calibration of the beam displacements used in ISR luminosity measurements", CERN-ISR-ES-BOM-82-15, 1982.

[4] K. A. Drees and S. M. White, "Vernier Scan Results from the First RHIC Proton Run at 250 GeV", proceedings of IPAC'10, Kyoto, Japan, May 2010.

[5] M. Ferro-Luzzi, "Determination of the luminosity at the LHC experiments", proceedings of ICHEP'10, Paris, July 2010, published in PoS (ICHEP 2010) 010;

S. M. White et al., "First Luminosity Scans in the LHC", proceedings of IPAC'10, Kyoto, Japan, May 2010.

[6] M. Ferro-Luzzi, Nucl. Instrum. Methods A 553, 388 (2005).

[7] R. Aaij et al, LHCb collaboration, Phys. Lett. B 693, 69 (2010), Phys. Lett. B 694, 209 (2010). 